\title{
Scrambling in two-dimensional conformal field theories with light and smeared operators
}

\author{
Harsha R. Hampapura, ${ }^{1, *}$ Andrew Rolph, ${ }^{1,2, \dagger}$ and Bogdan Stoica ${ }^{1,3, *}$ \\ ${ }^{1}$ Martin A. Fisher School of Physics, Brandeis University, Waltham, Massachusetts 02453, USA \\ ${ }^{2}$ Kavli Institute of Theoretical Physics, University of California, Santa Barbara, California 93106, USA \\ ${ }^{3}$ Department of Physics, Brown University, Providence, Rhode Island 02912, USA
}

(Received 21 December 2018; published 20 May 2019)

\begin{abstract}
We study quantum chaos in two-dimensional conformal field theories, building on the work analyzing the out-of-time order thermal correlation functions using large- $c$ Virasoro blocks. Our work investigates the contribution of light intermediate channels and smearing length scales to the four-point function and scrambling. Precise relations for how light intermediate channels increase the scrambling time and how smearing length scales smaller than the thermal length scale decrease the scrambling time are derived.
\end{abstract}

DOI: $10.1103 /$ PhysRevD.99.106010

\section{INTRODUCTION}

Unitary quantum mechanical systems do not exhibit information loss in that they never forget their initial state, however observers without access to all degrees of freedom (d.o.f.) may not be able to distinguish microstates. If an observer with access to less than half the system's d.o.f. cannot distinguish a perturbed state from an unperturbed one then the information contained in the perturbation is said to be scrambled. The time required for this dynamical chaotic mixing to occur is the scrambling time.

In classical physics chaos is understood as sensitivity to initial conditions. If one considers the phase space coordinate $x(t)$ in a classical chaotic system and perturbs $x(0)$ an infinitesimal amount, one can diagnose the sensitivity to initial conditions through the Poisson bracket

$$
\{x(t), p(0)\}=\frac{\partial x(t)}{\partial x(0)} .
$$

This quantity initially grows as a sum of exponentials in $t$, with the exponents called Lyapunov exponents. An analogous quantity to consider for a quantum system in state $\rho$ is the squared commutator [1],

$$
C(t):=-\operatorname{Tr}\left(\rho[W(t), V(0)]^{2}\right) .
$$

At the onset of scrambling, $C(t)$ also grows exponentially with $t$, which from the perspective of operator growth arises

\footnotetext{
*hrharsha@brandeis.edu

†andrewrolph@brandeis.edu

bstoica@brandeis.edu
}

Published by the American Physical Society under the terms of the Creative Commons Attribution 4.0 International license. Further distribution of this work must maintain attribution to the author(s) and the published article's title, journal citation, and DOI. Funded by SCOAP ${ }^{3}$. from how the unitarily evolved $W(t)=e^{i H t} W(0) e^{-i H t}$ grows with time to become a larger sum of longer operator products, due to nontrivial commutations with the Hamiltonian. Loosely speaking, the commutator squared is determined by the fraction of operator products in $W(t)$ which contain $V(0)$.

The systems we are especially interested in are many-body systems in thermal equilibrium, partly due to the AdS/CFT correspondence [2] and the holographic dual description of black holes as thermofield double (TFD) states in double copies of large $c$ CFTs [3]. For holographic field theories one can understand scrambling in thermal states as a disruption of the special TFD state entanglement between the left and right CFTs as diagnosed by mutual information between subregions in the two copies, which on the bulk side corresponds to the lengthening of the wormhole connecting the two asymptotic regions [4-7]. The wormhole lengthens because low energy quanta produced far in the past become highly boosted near the black hole horizon, giving a large shockwave backreaction to the geometry.

Scrambling is also relevant to the black hole information paradox, where a remarkable result [8] shows that, in certain cases, information absorbed by a black hole can be emitted almost immediately after it has been scrambled amongst the black hole d.o.f. It has been conjectured $[9,10]$ that black holes scramble information faster than any other quantum system in nature, and some evidence for this conjecture has been found [11].

Returning to our tool for studying quantum chaos, the squared commutator $C(t)$, expanding out gives four terms:

$$
\begin{aligned}
C(t)= & \langle V(0) W(t) W(t) V(0)+W(t) V(0) V(0) W(t) \\
& -W(t) V(0) W(t) V(0)-V(0) W(t) V(0) W(t)\rangle_{\beta} .
\end{aligned}
$$


This simplifies after the thermal relaxation time as $V$ acting on the thermal state becomes indistinguishable from the thermal state to local operators. The term $\langle V W W V\rangle_{\beta}$ can be understood as the expectation value of the operator $W W$ in the state obtained by $V$ acting on the thermal ensemble (and vice versa for $\langle W V V W\rangle_{\beta}$ ). If the energy inserted by the operator $V$ is small then after the dissipation time $\langle V W W V\rangle_{\beta}$ is given by the thermal expectation value $\langle W W\rangle_{\beta}$ multiplied by the norm of the state $\langle V V\rangle_{\beta}$. Thus, Eq. (1.3) becomes

$$
C(t)=2\left[\langle W(t) W(t)\rangle_{\beta}\langle V(0) V(0)\rangle_{\beta}-\Re\{\operatorname{OTOC}(t)\}\right],
$$

with the out-of-time ordered correlator (OTOC) defined by

$$
\operatorname{OTOC}(t):=\langle W(t) V(0) W(t) V(0)\rangle_{\beta} .
$$

OTOCs and $C(t)$ are thus equivalent ways of diagnosing chaos. At early times, the disconnected product and the OTOC terms in Eq. (1.4) cancel and the commutator squared is zero. At the onset of scrambling, the OTOC decays at a bounded rate $\lambda_{L} \leq 2 \pi / \beta[12-14]$. We define scrambling time as the operator time separation $t$ at which the OTOC is exponentially decaying to zero, or equivalently when $C(t)$ is asymptotically approaching the disconnected product $2\langle V V\rangle\langle W W\rangle$.

There is a significant body of literature on understanding scrambling from the holographic bulk perspective. In contrast, we will do a purely field-theoretic study, primarily building on work in [15], though we will give some holographic interpretation in the Discussion. Thermalization and scrambling in 2D CFTs has been previously studied through the time evolution of entanglement entropy [16-22]. The authors of [15] instead calculate an OTOC, using the known form of semiclassical Virasoro blocks for heavy-light operators [23]. Specifically they consider the contribution of the identity block to the OTOC, and from it, extract the scrambling time

$$
t_{*}=\frac{\beta}{2 \pi} \log \frac{c}{h_{w}},
$$

with $h_{w}$ the holomorphic weight of the $W$ operator. Ideally, one would analyze scrambling for a pair of light operators with both $h_{v}, h_{w} \ll c$, however the formula used for the semiclassical conformal block is only valid for fixed $h_{w} / c$, corresponding to a heavy $W$ operator. In [24], by matching with a bulk shockwave calculation, the authors conjecture the validity of the semiclassical formula for light-light operators.

In this paper, we investigate the effect of nonidentity Virasoro blocks and of smearing length scales on the scrambling time (1.6). We examine the contribution of higher primaries and demonstrate that the scrambling time depends on the spectrum of the CFT, and that the existence of a light primary operator with $\mathcal{O}(1)$ operator product expansion (OPE) coefficients with the $V$ and $W$ operators, and conformal weights $h_{p}, \bar{h}_{p} \ll c$ bounds the scrambling time from below as

$$
t_{*} \geq \frac{\beta}{2 \pi}\left(\frac{2 h_{v}+h_{p}}{2 h_{v}+\bar{h}_{p}}\right) \log \left(\frac{c}{h_{w}}\right),
$$

with $h_{v}$ the holomorphic weight of the $V$ operator. The scrambling time is determined by the primary operator for which the prefactor in (1.7) is the largest. Light operators with $h_{p}>\bar{h}_{p}$ increase the scrambling time. Note that primary operators with $\bar{h}_{p}>h_{p}$ do not violate the fast scrambling conjecture, as the existence of the identity operator bounds the scrambling time from below by (1.6).

We also consider the scrambling of operator-valued distributions, smearing the $V$ and $W$ operators over spatial scales $L_{V}, L_{W}$ in order to better understand the relation between the energy scale of perturbations and scrambling time. We calculate the scrambling time for smearing length scales much smaller than the thermal length scale to be

$$
t_{*}=\frac{\beta}{2 \pi}\left(\log \left(\frac{c}{h_{w}}\right)-\log \left(\frac{\beta^{2}}{L_{V} L_{W}}\right)\right),
$$

corresponding to a reduction in scrambling time due to high energy modes. For smearing length scales greater than $\beta$ we argue that the scrambling time increases without limit. The scrambling time (1.6) is the intermediate regime for perturbations with thermal scale energy.

The plan of this paper is as follows: In Sec. II, we set up the necessary CFT conventions and formulas to analyze the OTOC, in Sec. III we use the semiclassical Virasoro block to calculate the scrambling time in large $c$ two-dimensional CFTs with light higher-spin primaries. In Sec. IV we calculate how the spatial smearing of the operators $V$ and $W$ changes the scrambling time, and in Sec. V we discuss the holographic interpretation of our results and future directions.

Note.-This paper has some overlap in scope with work [25], as the two sets of authors worked in collaboration for much of the project until deciding to publish separately. A discussion of differences in analysis, results and interpretation is given in the Appendix.

\section{CFT BACKGROUND AND CONVENTIONS}

We are interested in the thermal four-point correlation function involving two operators $V$ and $W$ separated by Lorentzian time $t$ and spatial distance $x$. Correlation functions in the thermal state can be mapped to expectation values in the vacuum using the conformal transformation;

$$
z(x, t)=e^{\frac{2 \pi}{\beta}(x+t)}, \quad \bar{z}(x, t)=e^{\frac{2 \pi}{\beta}(x-t)},
$$


with correlators of primary operators related by

$$
\left\langle\mathcal{O}_{h, \bar{h}}(x, t) \ldots\right\rangle_{\beta}=\left(\frac{2 \pi z}{\beta}\right)^{h}\left(\frac{2 \pi \bar{z}}{\beta}\right)^{\bar{h}}\left\langle\mathcal{O}_{h, \bar{h}}(z, \bar{z}) \ldots\right\rangle .
$$

The specific correlation function we wish to work with is a four-point function, normalized by the product of twopoint functions,

$$
\frac{\left\langle W\left(z_{1}, \bar{z}_{1}\right) W\left(z_{2}, \bar{z}_{2}\right) V\left(z_{3}, \bar{z}_{3}\right) V\left(z_{4}, \bar{z}_{4}\right)\right\rangle}{\left\langle W\left(z_{1}, \bar{z}_{1}\right) W\left(z_{2}, \bar{z}_{2}\right)\right\rangle\left\langle V\left(z_{3}, \bar{z}_{3}\right) V\left(z_{4}, \bar{z}_{4}\right)\right\rangle} .
$$

Following the canonical choice, we use the global conformal transformations $\operatorname{SL}(2, \mathbb{C})$ to take $z_{1}, \bar{z}_{1} \rightarrow \infty ; z_{2}$, $\bar{z}_{2} \rightarrow 1 ; z_{4}, \bar{z}_{4} \rightarrow 0$ [26]. With this choice, the holomorphic cross ratio is

$$
z:=\frac{z_{12} z_{34}}{z_{13} z_{24}}=z_{3}
$$

and similarly $\bar{z}=\bar{z}_{3}$, and the ratio (2.3) becomes

$$
\begin{aligned}
& \frac{\left\langle W\left(z_{1}, \bar{z}_{1}\right) W\left(z_{2}, \bar{z}_{2}\right) V\left(z_{3}, \bar{z}_{3}\right) V\left(z_{4}, \bar{z}_{4}\right)\right\rangle}{\left\langle W\left(z_{1}, \bar{z}_{1}\right) W\left(z_{2}, \bar{z}_{2}\right)\right\rangle\left\langle V\left(z_{3}, \bar{z}_{3}\right) V\left(z_{4}, \bar{z}_{4}\right)\right.} \\
& =\lim _{z_{1}, \bar{z}_{1} \rightarrow \infty} z_{1}^{2 h_{w}} \bar{z}_{1}^{2 \bar{h}_{w}} z^{2 h_{v}} \bar{z}^{2 \bar{h}_{v}}\left\langle W\left(z_{1}, \bar{z}_{1}\right) W(1,1) V(z, \bar{z}) V(0,0)\right\rangle \\
& =\sum_{p} C_{V V}^{p} C_{W W}^{p} z^{2 h_{v}} \mathcal{F}_{p}(z) \bar{z}^{2 \bar{h}_{v}} \mathcal{F}_{p}(\bar{z})
\end{aligned}
$$

where, in the final line, we have expanded in the $z \rightarrow 0$ channel to write the four-point function in terms of the Virasoro conformal blocks. From Eq. (2.5) we see that the quantity of interest when comparing the OTOC to the disconnected product is $z^{2 h_{v}} \mathcal{F}_{p}(z)$ and its antiholomorphic counterpart. ${ }^{1}$

\section{LIGHT INTERMEDIATE CHANNELS}

In this section we investigate the importance of intermediate channels to the OTOC, their dominance over the identity block and subsequent effect on the scrambling time. In putative bulk theories, this corresponds to the exchange of massive particles. Following [15], we will work in the semiclassical limit $c \gg 1$ where the conformal blocks $\mathcal{F}_{p}(z)$ exponentiate,

$$
\mathcal{F}_{p}(z)=\exp \left(-\frac{c}{6} f_{p}(z)\right)
$$

and use a result from [23] for the semiclassical conformal block, valid for $c \gg 1 ; h_{v}, h_{p} \ll c ; h_{w} / c$ fixed but arbitrary,

\footnotetext{
${ }^{1}$ The factor of $z^{2 h_{v}}$ agrees with the convention of [24] and is nonstandard.
}

$$
\begin{aligned}
\frac{c}{6} f(z)= & \left(2 h_{v}-h_{p}\right) \log \left(\frac{1-(1-z)^{\alpha_{w}}}{\alpha_{w}}\right) \\
& +h_{v}\left(1-\alpha_{w}\right) \log (1-z) \\
& +2 h_{p} \log \left(\frac{1+(1-z)^{\alpha_{w} / 2}}{2}\right)
\end{aligned}
$$

with $\alpha_{w}:=\sqrt{1-24 h_{w} / c}$. This gives a conformal block

$$
\begin{aligned}
z^{2 h_{v}} \mathcal{F}_{p}(z)= & {\left[\frac{\alpha_{w} z(1-z)^{\left(\alpha_{w}-1\right) / 2}}{1-(1-z)^{\alpha_{w}}}\right]^{2 h_{v}} } \\
& \cdot\left[\frac{4}{\alpha_{w}} \frac{1-(1-z)^{\alpha_{w} / 2}}{1+(1-z)^{\alpha_{w} / 2}}\right]^{h_{p}} .
\end{aligned}
$$

Following the procedure detailed in [15] we consider the analytic continuation of the Euclidean four-point function to Lorentzian time which gives the OTOC's ordering of operators. This analytic continuation causes $z$ to pass around its branch point at $z=1$ and hence for $z^{2 h_{v}} \mathcal{F}_{p}(z)$ to pass to the second Riemann sheet. $\bar{z}$ does not pass around its branch point at $\bar{z}=1$. The conformal block on the second sheet is

$$
\begin{aligned}
z^{2 h_{v}} \mathcal{F}_{p}^{I I}(z)= & {\left[\frac{\alpha_{w} z e^{-\pi\left(\alpha_{w}-1\right) i}(1-z)^{\left(\alpha_{w}-1\right) / 2}}{1-e^{-2 \pi i \alpha_{w}}(1-z)^{\alpha_{w}}}\right]^{2 h_{v}} } \\
& \cdot\left[\frac{4}{\alpha_{w}} \frac{1-e^{-\pi \alpha_{w} i}(1-z)^{\alpha_{w} / 2}}{1+e^{-\pi \alpha_{w} i}(1-z)^{\alpha_{w} / 2}}\right]^{h_{p}} .
\end{aligned}
$$

We are interested in the behavior of the conformal block in three different time regimes: before the fast scrambling time (1.6) but after the dissipation time $t \sim \beta$, around the fast scrambling time, and after the fast scrambling time, corresponding to $h_{w} / c \ll z \ll 1, z \sim h_{w} / c$, and $z \ll h_{w} / c$ respectively. In these three regimes the conformal block takes the form

$$
z^{2 h_{v}} \mathcal{F}_{p}^{I I}(z) \approx \begin{cases}\left(\frac{16}{z}\right)^{h_{p}} & h_{w} / c \ll z \ll 1 \\ \left(\frac{16}{z}\right)^{h_{p}}\left(\frac{1}{1-\frac{24 \pi i h_{w}}{c z}}\right)^{2 h_{v}+h_{p}} & z \sim h_{w} / c \\ 16^{h_{p}}\left(\frac{i c}{24 \pi h_{w}}\right)^{2 h_{v}+h_{p}} z^{2 h_{v}} & z \ll h_{w} / c .\end{cases}
$$

To see how this function depends on $t$ recall that $z \sim e^{-\frac{2 \pi}{\beta} t}$. Figure 1 illustrates how the conformal block depends on the operator time separation for a few values of $h_{p}$. For the identity block with $h_{p}=0$ it is constant at early times when $z \gg h_{w} / c$, then starts to exponentially decay around $z \sim h_{w} / c$. The light intermediate channels have significantly different behavior, they initially grow exponentially with $t$ and then start to decay roughly around the same time as the identity block. 


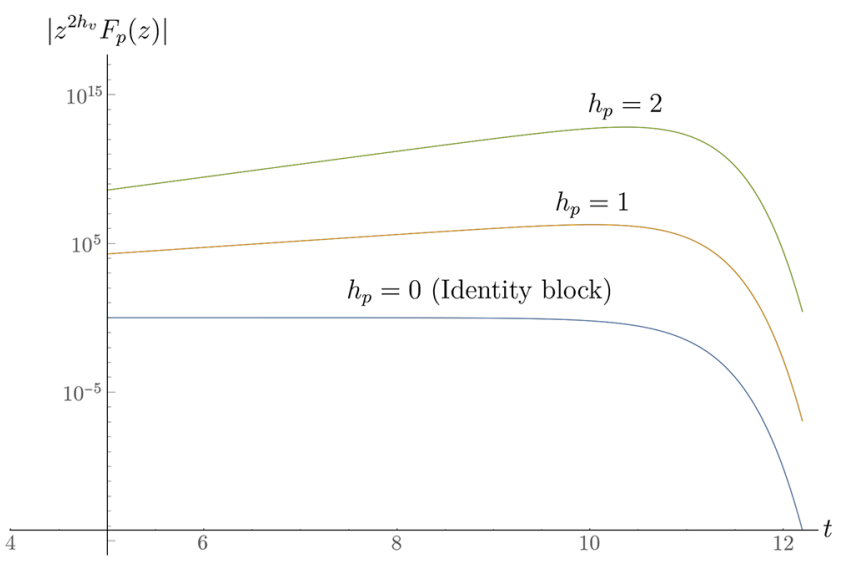

FIG. 1. Conformal block $\left|z^{2 h_{v}} \mathcal{F}_{p}^{I I}(z)\right|$ against time separation $t$ for different values of intermediate channel holomorphic weight $h_{p}$, for $h_{w}=5, h_{v}=1000$ and $c=10^{9}$. The figure shows the initial exponential growth with $t$ of the conformal block with $h_{p}>0$, the decay around scrambling time, and the dominance of light intermediate conformal blocks over the identity block.

For the OTOC, one also needs to consider the effect of the antiholomorphic conformal block factor $\bar{z}^{2 \bar{h}_{v}} \mathcal{F}_{p}(\bar{z})$. The key difference from the holomorphic block is that during the analytic continuation of the Euclidean four-point function, the holomorphic cross ratio $z$ passes around its branch point at $z=1$ and goes to the second Riemann sheet, while the antiholomorphic cross ratio does not and stays on the principal sheet. The principal sheet antiholomorphic block acts to suppress the exponential growth of the second sheet holomorphic block, as seen by replacing holomorphic variables in (3.3) with their antiholomorphic counterparts, then taking $\bar{z} \ll 1$,

$$
\bar{z}^{2 \bar{h}_{v}} \mathcal{F}_{p}(\bar{z}) \approx(2 \bar{z})^{\bar{h}_{p}} \propto \exp \left(-\frac{2 \pi}{\beta} \bar{h}_{p}(t+x)\right) .
$$

For the identity block with $\bar{h}_{p}=0$ this factor is trivial and irrelevant to the scrambling time derivation in [24]. However, for $\bar{h}_{p}>0$, this factor acts to suppress the contribution of light primary operators, it is an exponentially decaying function in $t$. We will discuss the case $\bar{h}_{p}<0$ after combining this antiholomorphic conformal block with its second sheet holomorphic counterpart, to find the behavior of the OTOC with $t$.

At early times, though with $t \gg \beta$, the product of conformal blocks grows exponentially with $t$ as

$$
z^{2 h_{v}} \mathcal{F}_{p}^{I I}(z) \bar{z}^{2 \bar{h}_{v}} \mathcal{F}_{p}(\bar{z}) \propto \exp \left(\frac{2 \pi}{\beta}\left(h_{p}-\bar{h}_{p}\right) t\right),
$$

and surprisingly, this contribution to the OTOC dominates over the identity block at $t$ given by (1.6), assuming $h_{p}>\bar{h}_{p}$ and generic OPE coefficients (We shall make this condition more precise later.) Around this time the second expression in (3.5) shows that all the holomorphic conformal blocks switch from exponential growth in $t$ to exponential decay. The exception is the identity block as $\left|z^{2 h_{v}} \mathcal{F}_{0}^{I I}(z)\right|$ is a monotonically decreasing function in time, starting at 1 before starting its exponential decay as scrambling takes effect. The holomorphic conformal blocks for the other primary operators reach their maximum magnitude at

$$
z_{\max }\left(h_{p}\right)=2 \pi\left(1-\alpha_{w}\right) \sqrt{\frac{2 h_{v}}{h_{p}}},
$$

corresponding to a time separation

$$
t_{\max }=\frac{\beta}{2 \pi} \log \left(\sqrt{\frac{h_{p}}{h_{v}}} \frac{c}{h_{w}}\right) .
$$

At late times, all holomorphic blocks decay at the same rate determined by the third equation in (3.5), $\exp \left(-\frac{4 \pi}{\beta} h_{v} t\right)$, and so the light intermediate states dominate over the identity block for all times with no crossover. Including the contribution of the antiholomorphic block gives

$z^{2 h_{v}} \mathcal{F}_{p}^{I I}(z) \bar{z}^{2 \bar{h}_{v}} \mathcal{F}_{p}(\bar{z}) \sim\left(\frac{c}{h_{w}}\right)^{2 h_{v}+h_{p}} \exp \left(-\left(2 h_{v}+\bar{h}_{p}\right) \frac{2 \pi}{\beta} t\right)$

at late times, when $z \ll h_{w} / c$. What we are most interested in is the value of $t$ for which the commutator squared $C(t)$ approaches $2\langle W(t) W(t)\rangle_{\beta}\langle V(0) V(0)\rangle_{\beta}$, or equivalently when the $\mid$ OTOC $\mid \ll 1$. The time taken for $z^{2 h_{v}} \mathcal{F}_{p}^{I I}(z) \bar{z}^{2 \bar{h}_{v}} \mathcal{F}_{p}(\bar{z})$ to decay to an $\mathcal{O}(1)$ value is

$$
t_{* p}=\frac{\beta}{2 \pi}\left(\frac{2 h_{v}+h_{p}}{2 h_{v}+\bar{h}_{p}}\right) \log \left(\frac{c}{h_{w}}\right) .
$$

Assuming OPE coefficients that are not parametrically small in powers of $h_{w} / c$ in order to suppress the contribution of light primary operators, the scrambling time is determined by the primary operator for which the prefactor in (3.11) is largest, as the OTOC is dominated by the conformal block for that operator. Roughly speaking, the larger the spin the longer the scrambling time. As explained in the Introduction, the existence of primary operators with $\bar{h}_{p}>h_{p}$ does not lead to a shorter scrambling time, the scrambling time in the 2D CFTs we are considering is bounded from below by (1.6). We also note that at times much later than (3.11), including the contribution of both holomorphic and antiholomorphic blocks (3.10) implies that the block with a larger value of $\bar{h}_{p}$ decays faster and there is a crossover. In particular, the identity block dominates over all other blocks at sufficiently late times. 
The apparent asymmetry between $h_{p}$ and $\bar{h}_{p}$ occurs because we are looking at Lorentzian correlators and this breaks the symmetry between holomorphic and antiholomorphic sectors of the CFT. In parity invariant CFTs, where for each primary operator with $\left(h_{p}, \bar{h}_{p}\right)$ there is a conjugate operator with weights $\left(\bar{h}_{p}, h_{p}\right)$, the scrambling time is parity invariant. While the decay time for a given block, given by (3.11), is not invariant under $h_{p} \leftrightarrow \bar{h}_{p}$, the OTOC from which the scrambling time is derived is a sum over the full spectrum, which will be parity invariant. Assuming the parity invariant CFT has light operators and generic OPE coefficients, the scrambling time is increased.

Finally, we want to note that our assumption of generic OPE coefficients can be made more precise. Demanding that the contribution of the identity block be less than the contribution of other light primaries, we get

$$
\begin{aligned}
& C_{V V}^{i} C_{W W}^{i} z^{2 h_{v}} \mathcal{F}_{i}^{I I}(z) \bar{z}^{2 \bar{h}_{v}} \mathcal{F}_{i}(\bar{z}) \\
& \quad<C_{V V}^{p} C_{W W}^{p} z^{2 h_{v}} \mathcal{F}_{p}^{I I}(z) \bar{z}^{2 \bar{h}_{v}} \mathcal{F}_{p}(\bar{z}),
\end{aligned}
$$

where $C_{V V}^{i}$ and $C_{W W}^{i}$ are the OPE coefficients associated with the identity block. We now use the second expression in (3.5) to maximize the ratio between the nonidentity and the identity blocks and find

$$
\frac{C_{V V}^{p} C_{W W}^{p}}{C_{V V}^{i} C_{W W}^{i}}>\left(\frac{h_{w}}{c}\right)^{h_{p}-h_{\bar{p}}}
$$

which gives a constraint on the ratios of OPE coefficients in terms of the $h_{w}, c$ and the spin of the intermediate primary. At present, we do not have a good intuition for how easy it is for a CFT to satisfy the constraint (3.13). We hope to address this interesting question in the future.

\section{SPATIALLY SMEARED OPERATORS}

In this section we will change gears and consider a different computation, in which we smear the operators over finite length scales. We would like to understand the effect of these scales on the scrambling time, to see how scrambling depends on the perturbations' energies. Let us introduce our setup. The first step in the smearing procedure is to consider point operators that are not spatially coincident and then integrate against smearing functions. After the smearing procedure, each operator in a given pair will have finite spatial support and be centered about the same spatial position. Our labeling of the four operator positions is shown in Fig. 2. After the conformal mapping given by

$$
\begin{array}{llrl}
z_{1}=e^{\frac{2 \pi}{\beta}\left(x_{1}+t\right)}, & \bar{z}_{1}=e^{\frac{2 \pi}{\beta}\left(x_{1}-t\right)} \\
z_{2}=e^{\frac{2 \pi}{\beta}\left(x_{2}+t\right)}, & \bar{z}_{2}=e^{\frac{2 \pi}{\beta}\left(x_{2}-t\right)} \\
z_{3}=e^{\frac{2 \pi}{\beta} x_{3}}, & \bar{z}_{3}=e^{\frac{2 \pi}{\beta} x_{3}} \\
z_{4}=e^{\frac{2 \pi}{\beta} x_{4}}, & \bar{z}_{4}=e^{\frac{2 \pi}{\beta} x_{4}},
\end{array}
$$

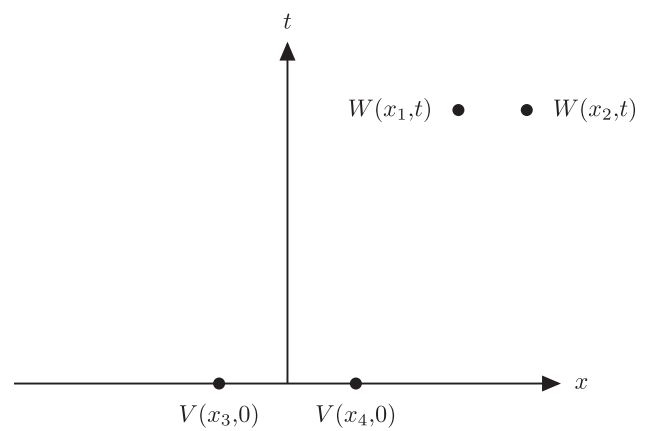

FIG. 2. Positions of the point $V$ and $W$ operators prior to integration against Gaussian smearing functions of width $L_{V}$ and $L_{W}$.

the holomorphic and antiholomorphic cross ratios $z=$ $z_{12} z_{34} / z_{13} z_{24}$ and $\bar{z}=\bar{z}_{12} \bar{z}_{34} / \bar{z}_{13} \bar{z}_{24}$ are

$$
\begin{aligned}
& z=\frac{\sinh \left(\frac{\pi}{\beta}\left(x_{1}-x_{2}\right)\right) \sinh \left(\frac{\pi}{\beta}\left(x_{3}-x_{4}\right)\right)}{\sinh \left(\frac{\pi}{\beta}\left(t+x_{1}-x_{3}\right)\right) \sinh \left(\frac{\pi}{\beta}\left(t+x_{2}-x_{4}\right)\right)}, \\
& \bar{z}=\frac{\sinh \left(\frac{\pi}{\beta}\left(x_{1}-x_{2}\right)\right) \sinh \left(\frac{\pi}{\beta}\left(x_{3}-x_{4}\right)\right)}{\sinh \left(\frac{\pi}{\beta}\left(t-x_{1}+x_{3}\right)\right) \sinh \left(\frac{\pi}{\beta}\left(t-x_{2}+x_{4}\right)\right)} .
\end{aligned}
$$

We take $t$ to be much larger than any $x_{i}$, otherwise the $V$ and $W$ operators have support on spacelike separated regions and by causality those components of the operators commute. In this limit the cross ratio becomes

$$
\begin{aligned}
z= & 4 \sinh \left(\frac{\pi}{\beta}\left(x_{1}-x_{2}\right)\right) \sinh \left(\frac{\pi}{\beta}\left(x_{3}-x_{4}\right)\right) \\
& \times e^{-\frac{2 \pi t}{\beta} t} e^{\frac{\pi}{\beta}\left(-x_{1}-x_{2}+x_{3}+x_{4}\right)} .
\end{aligned}
$$

From (3.5) we recall that the second sheet identity block is

$$
z^{2 h_{v}} \mathcal{F}^{I I}(z) \approx\left(\frac{1}{1-\frac{24 \pi i h_{w}}{c z}}\right)^{2 h_{v}} .
$$

This has a dependence on the four operator spatial positions $x_{i}$, through the dependence of $z$ on $x_{i}$, this is what we integrate against our smearing functions. We integrate the point operators $V$ and $W$ against Gaussians smearing functions with spatial width $L_{V}$ and $L_{W}$. The $V$ operator will be centred around $x=0$ while the $W$ has a spatial offset, it is smeared about $x=x_{W}$, defined with the appropriate prefactor,

$$
W_{\text {smear }}\left(t, x_{W}\right):=\frac{1}{L_{W} \sqrt{\pi}} \int_{-\infty}^{\infty} e^{-\left(x-x_{W}\right)^{2} / L_{W}^{2}} W(t, x) d x .
$$

This is an operator at Lorentzian time $t$, smeared over a length scale $L_{W}$ about a central position $x_{W}$. The conformal block for the four smeared operators is then 


$$
\begin{aligned}
& \int_{-\infty}^{\infty} d x_{1} d x_{2} d x_{3} d x_{4} \frac{1}{L_{W}^{2} L_{V}^{2} \pi^{2}} \\
& \quad \times \exp \left(-\frac{x_{1}^{2}}{L_{V}^{2}}-\frac{x_{2}^{2}}{L_{V}^{2}}-\frac{\left(x_{3}-x_{W}\right)^{2}}{L_{W}^{2}}-\frac{\left(x_{4}-x_{W}\right)^{2}}{L_{W}^{2}}\right) z^{2 h_{v}} \mathcal{F}(z) .
\end{aligned}
$$

In terms of the integral, smearing length scale affects scrambling time because the parts of the integration region which contribute significantly depend on $L_{V}$ and $L_{W}$. Similar to the unsmeared identity block, we consider the perturbation to have been scrambled once the conformal block switches from being constant to exponential decaying with $t$. The nonGaussian part of the integrand, given by (4.5), has two asymptotic $z$ regimes: for early times with $z \gg h_{w} / c$ it is 1 , while for late times with $z \ll h_{w} / c$ it is

$$
z^{2 h_{v}} \mathcal{F}(z) \approx\left(\frac{c z}{24 \pi i h_{w}}\right)^{2 h_{v}} \ll 1 .
$$

Note that from (4.4) $z$ is small for late times, or when the separation of operators within a pair is much less than the thermal length scale. The smaller the smearing length scale, the more the Gaussian smearing functions suppress large $x_{i}$, and so the dominant contribution to the integral is for $z \ll h_{w} / c$ with the conformal block given by (4.8). For $L_{V}, L_{W} \ll \beta$ separations of operators within a pair of order the thermal length scale and larger are suppressed, and we can approximate the cross ratio (4.4) by

$$
z \approx \frac{4 \pi^{2}}{\beta^{2}}\left(x_{1}-x_{2}\right)\left(x_{3}-x_{4}\right) e^{-\frac{2 \pi}{\beta}\left(t-\frac{x_{3}+x_{4}}{2}\right)} .
$$

Combining (4.9) and (4.8) we can exactly evaluate the integral (4.7), giving the smeared conformal block

$$
\frac{1}{4 \pi}\left(\left(1+e^{2 \pi i h_{v}}\right) \Gamma\left(\frac{1}{2}+h_{v}\right)\right)^{2}\left(\frac{\pi c}{3 i h_{w}} \frac{L_{V} L_{W}}{\beta^{2}} e^{-\frac{2 \pi}{\beta}\left(t-x_{W}\right)}\right)^{2 h_{v}} .
$$

Note that if $h_{v}$ is half-integer then this expression is exactly zero, however in discussing scrambling we wish to consider generic operators $V$, for which $h_{v}$ will not be half-integer. The time separation at which (4.10) becomes $\mathcal{O}(1)$, marking the scrambling time, is

$$
t_{*}=\frac{\beta}{2 \pi}\left(\log \left(\frac{c}{h_{w}}\right)-\log \left(\frac{\beta^{2}}{L_{V} L_{W}}\right)\right),
$$

valid for $L_{V}, L_{W} \ll \beta$. The smaller the smearing length scale, the larger the energy scale of the operator and the faster the perturbation is scrambled. When $L_{V}$ and $L_{W}$ are sufficiently small they can decrease the scrambling time at leading order, however as these high energy perturbations are not a small perturbation to the thermal state this does not violate the fast scrambling conjecture.

For operators smeared over length scales much larger than the thermal length scale, the region of $\left\{x_{i}\right\} \in \mathbb{R}^{4}$ for which the Gaussians in (4.7) give support grows beyond the region of size $\beta^{4}$ centered around $\left(x_{1}, x_{2}, x_{3}, x_{4}\right)=\left(0,0, x_{W}, x_{W}\right)$, and the $z \gg h_{w} / c$ limit for which the conformal block is 1 becomes the important $z$ limit. The larger one takes $L_{V}$ and $L_{W}$, the larger the region in $\mathbb{R}^{4}$ which is both not suppressed by the Gaussians and has $z \gg h_{w} / c$, and so the closer the smeared conformal block is to one for fixed $t$. By increasing the smearing length scale one increases the scrambling time. This is valid until one one reaches smearing length scales as wide as the light cone. Interpolating between the result (4.11) for $L_{V}, L_{W} \ll \beta$ and the $L_{V}, L_{W} \gg \beta$ behavior just described to $L_{V}, L_{W} \sim \beta$ one concludes that operators smeared over the thermal length scale exhibit fast scrambling, at least when considering only the contribution of the identity block.

\section{DISCUSSION}

In this section we discuss the holographic interpretation of our results, the relation to the chaos bound, and possible future work.

Let us first give a holographic interpretation for the dependence of scrambling time on smearing length scale. Perturbing the thermal state of a holographic 2D CFT with a single trace operator smeared over spatial length $L$ is dual to releasing a particle of energy $E \sim L^{-1}$ from the asymptotic boundary of a Bañados-Teitelboim-Zanelli black hole. As we increase the smearing length scale, we reduce the energy of the bulk particle. Near the horizon, time translation corresponds to a boost, such that on the $t=0$ slice a particle of energy $E$ released at the boundary at time $t=-t_{w}$ has proper energy

$$
E_{p} \sim \frac{E \beta}{2 \pi l_{\mathrm{AdS}}} \exp \left(\frac{\beta}{2 \pi} t_{w}\right) .
$$

Scrambling occurs when $t_{w}$ is large enough that the proper energy becomes of order $G_{N}^{-1} \sim c$, then the backreaction on the Bañados-Teitelboim-Zanelli black hole geometry becomes significant, leading to the lengthening of the wormhole and destruction of entanglement between the two CFT copies. If we increase $L$ or equivalently decrease $E$ then it takes a longer time to scramble. Equation (5.1) suggests that the dependence of scrambling time on the smearing length of the $W$ operator is $(\beta / 2 \pi) \log \left(\beta / L_{W}\right)$, this is consistent with our result (4.11).

The dominating contribution of higher dimension and spin primaries to the scrambling time in the CFT corresponds to bulk to bulk propagation of massive spin fields between the $V$ and $W$ fields. Assuming that the twodimensional CFTs we have been studying that seem to have large scrambling times do in fact exist, and that they have a semiclassical quantum gravity dual, it is puzzling from the 
bulk perspective why the massive fields dual to the light intermediaries should increase the scrambling time.

The arbitrarily rapid exponential growth of the conformal block seen in Eq. (3.7) for $\beta \ll t \ll(\beta / 2 \pi) \log c$ does not violate the chaos bound. The chaos bound on Lyapunov exponents is derived by showing that functions $f(t)$ that are analytic and bounded $|f| \leq 1$ on the half-strip given by $\Re t>0,|\Im t| \leq \beta / 4$ satisfy the inequality

$$
\frac{1}{1-|f|}\left|\frac{d f}{d t}\right| \leq \frac{2 \pi}{\beta}+\mathcal{O}\left(\exp \left(-\frac{4 \pi}{\beta} t\right)\right),
$$

then assuming the ratio of the OTOC to the disconnected product is of the form

$$
\frac{\langle V W(t) V W(t)\rangle_{\beta}}{\langle V V\rangle_{\beta}\langle W(t) W(t)\rangle_{\beta}} \approx 1-\epsilon e^{\lambda_{L} t}+\cdots
$$

satisfy the above requirements for $f$, and one finds $\lambda_{L} \leq 2 \pi / \beta$. The conformal block for light primaries we study in Sec. III is not of this form and does not obey the assumptions made in deriving the chaos bound. It is unusual in that it grows rather than decays with $t$, at an arbitrarily fast rate and to a value exponentially larger than the disconnected product. The chaos bound does not constrain the growth of the OTOC, so there is no inconsistency. That said, the conformal block is still a bounded function in $t$ and assuming it is analytic and bounded on the whole half-strip its rate of decay is bounded after reaching its maximum.

The early growth of the OTOC seems strange, as one usually interprets the decay of the OTOC as the onset of chaos, growth seems to imply increasing order in a chaotic system. Moreover, there is an argument that in large $N$ systems one expects the OTOC to be parametrically close to the disconnected product for all $t \gg \beta$, as detailed in Sec. 4.3.1 of [12]. In contrast, we found that each conformal block with $h_{p}>\bar{h}_{p}$ is exponentially larger than the disconnected product until long after the fast scrambling time. We are unclear as to the resolution of this apparent tension. It would be interesting to investigate if including heavy primaries makes the sum over Virasoro blocks divergent, and so necessitates a resummation.

In Sec. III we considered only light primaries with $\Delta \ll c$, but it is interesting to consider what effect primary operators of dimension $\Delta \sim c$ and heavier have on the OTOC and scrambling time. This question can partially be answered using the known semiclassical conformal blocks formula in the limit $h_{p} \rightarrow \infty$ with $c / h_{p}, h_{w} / c$ and $h_{v} / c$ all small and fixed [27], however we are not aware of formulas for intermediate weight primaries $h_{p} \sim c$.

A natural extension of our analysis is the study of the OTOC in higher dimensional CFTs. Although some results are available for a class of interesting models [28], a general analysis of the Virasoro blocks is rendered difficult by the absence of semiclassical results for the conformal blocks (which can be attributed to the presence of only global descendants). We leave this interesting question for future work.

\section{ACKNOWLEDGMENTS}

A. R., H. R. H., and B. S. would like to thank Matthew Headrick, Albion Lawrence, Eric Perlmutter, and Douglas Stanford for useful feedback on early versions of this draft, and Chang Liu and David Lowe for early collaboration. This work was supported in part by the U.S. Department of Energy under Grant No. DE-SC0009987, the National Science Foundation under Grant No. NSF PHY1748958, and by the Simons Foundation through the It from Qubit: Simons Collaboration on Quantum Fields, Gravity and Information. A. R. is also supported by a Kavli Institute for Theoretical Physics Graduate Fellowship, is thankful to the Kavli Institute of Theoretical Physics for hospitality, and to Adam Levine for useful discussions. This research was supported in part by the National Science Foundation under Grant No. NSF PHY-1748958. H. R. H. would like to thank Pawel Caputa, Sunny Guha, Sunil Mukhi and Pranjal Nayak for useful discussions. B.S. thanks Jennifer Lin for discussions.

\section{APPENDIX: REMARKS ON REF. [25]}

Here we note some differences in our paper compared to [25]. Our papers overlap in scope in that they study the effect of smeared operators and higher primaries on scrambling time. Let us highlight some differences in analysis and results to aid readers wishing to understand the results in the two papers.

(i) With regards the light primaries, our scrambling time $t_{* p}$ is not related to the $t_{* p}$ given in Eq. (12) of [25]. Our $t_{* p}$, for the dominating primary operator, corresponds to the time at which the commutator squared will approach the disconnected product $2\langle V V\rangle\langle W(t) W(t)\rangle$. In [25] the $t_{* p}$ computed is the time at which the ratio of the light primary operator block to the identity block saturates to a constant, which is not directly related to the scrambling time. The authors of [25] also did not consider the contribution of the antiholomorphic conformal block to the OTOC.

(ii) Our smearing analysis also differs from that in [25]. We smear each operator over its own Gaussian wave packet. Reference [25] smears one $V$ operator over a half-Gaussian on the positive $x$-axis, one over a mirror image half-Gaussian on the negative $x$-axis, and similarly for the $W$ operators. Varying $L$ then convolutes two separate effects on scrambling time, operator smearing length scale and spatial offset. 
[1] A. Kitaev, Hidden correlators in the Hawking radiation and thermal noise, Proceedings of the Fundamental Physics Prize Symposium, 2014.

[2] J. M. Maldacena, The large N limit of superconformal field theories and supergravity, Int. J. Theor. Phys. 38, 1113 (1999); Advances in theoretical and mathematical physics, Adv. Theor. Math. Phys. 2, 231 (1998).

[3] J. Maldacena, Eternal black holes in anti-de Sitter, J. High Energy Phys. 03 (2003) 021.

[4] S. H. Shenker and D. Stanford, Multiple shocks, J. High Energy Phys. 12 (2014) 046.

[5] S. H. Shenker and D. Stanford, Black holes and the butterfly effect, J. High Energy Phys. 03 (2014) 067.

[6] S. Leichenauer, Disrupting entanglement of black holes, Phys. Rev. D 90, 046009 (2014).

[7] S. H. Shenker and D. Stanford, Stringy effects in scrambling, J. High Energy Phys. 05 (2015) 132.

[8] P. Hayden and J. Preskill, Black holes as mirrors: Quantum information in random subsystems, J. High Energy Phys. 07 (2007) 120.

[9] Y. Sekino and L. Susskind, Fast scramblers, J. High Energy Phys. 10 (2008) 065.

[10] L. Susskind, Addendum to fast scramblers, arXiv:1101.6048.

[11] N. Lashkari, D. Stanford, M. Hastings, T. Osborne, and P. Hayden, Towards the fast scrambling conjecture, J. High Energy Phys. 04 (2013) 022.

[12] J. Maldacena, S. H. Shenker, and D. Stanford, A bound on chaos, J. High Energy Phys. 08 (2016) 106.

[13] A. L. Fitzpatrick and J. Kaplan, A quantum correction to chaos, J. High Energy Phys. 16 (2016) 70.

[14] E. Perlmutter, Bounding the space of holographic cfts with chaos, J. High Energy Phys. 16 (2016) 69.

[15] D. A. Roberts and D. Stanford, Diagnosing Chaos Using Four-Point Functions in Two-Dimensional Conformal Field Theory, Phys. Rev. Lett. 115, 131603 (2015).

[16] H. Liu and S. J. Suh, Entanglement Tsunami: Universal Scaling in Holographic Thermalization, Phys. Rev. Lett. 112, 011601 (2014).
[17] H. Liu and S. J. Suh, Entanglement growth during thermalization in holographic systems, Phys. Rev. D 89, 066012 (2014).

[18] V. Balasubramanian, A. Bernamonti, N. Copland, B. Craps, and F. Galli, Thermalization of mutual and tripartite information in strongly coupled two dimensional conformal field theories, Phys. Rev. D 84, 105017 (2011).

[19] C. T. Asplund and A. Bernamonti, Mutual information after a local quench in conformal field theory, Phys. Rev. D 89, 066015 (2014).

[20] T. Hartman and J. Maldacena, Time evolution of entanglement entropy from black hole interiors, J. High Energy Phys. 05 (2013) 014.

[21] A. Mollabashi, M. Nozaki, S. Ryu, and T. Takayanagi, Holographic geometry of cmera for quantum quenches and finite temperature, J. High Energy Phys. 03 (2014) 098.

[22] V. Balasubramanian, A. Bernamonti, J. de Boer, N. Copland, B. Craps, E. Keski-Vakkuri, B. Müller, A. Schäfer, M. Shigemori, and W. Staessens, Thermalization of Strongly Coupled Field Theories, Phys. Rev. Lett. 106, 191601 (2011).

[23] A. L. Fitzpatrick, J. Kaplan, and M. T. Walters, Universality of long-distance AdS physics from the CFT bootstrap, J. High Energy Phys. 08 (2014) 145.

[24] D. A. Roberts, D. Stanford, and L. Susskind, Localized shocks, J. High Energy Phys. 03 (2015) 051.

[25] C. Liu and D. A. Lowe, Notes on scrambling in conformal field theory, Phys. Rev. D 98, 126013 (2018)

[26] P. Di Francesco, P. Mathieu, and D. Senechal, Conformal Field Theory. Graduate Texts in Contemporary Physics (Springer-Verlag, New York, 1997).

[27] A. B. Zamolodchikov, Conformal symmetry in twodimensional space: Recursion representation of conformal block, Theor. Math. Phys. 73, 1088 (1987).

[28] D. Chowdhury and B. Swingle, Onset of many-body chaos in the O (N) model, Phys. Rev. D 96, 065005 (2017). 